\title{
Human cases of simultaneous echinococcosis and tuberculosis - significance and extent in China
}

\author{
Yu Rong Yang1,2,3, Darren J Gray ${ }^{2}$, Magda K Ellis ${ }^{2}$, Shu Kun Yang4, \\ Philip S Craig 5 and Donald P McManus*2
}

\begin{abstract}
Address: ${ }^{1}$ Ningxia Medical University, Yinchuan, Ningxia Hui Autonomous Region, PR China, ${ }^{2}$ Molecular Parasitology Laboratory, Queensland Institute of Medical Research, Brisbane, Australia, ${ }^{3}$ School of Population Health, University of Queensland, Brisbane, Australia, ${ }^{4}$ Teaching Hospital of Ningxia Medical University, Yinchuan, Ningxia Hui Autonomous Region, PR China and ${ }^{5}$ Biomedical Sciences Research Institute and School of Environment and Life Sciences, University of Salford, Salford, UK
\end{abstract}

Email: Yu Rong Yang - yangyurong@hotmail.com; Darren J Gray - darrenG@qimr.edu.au; Magda K Ellis - magdaE@qimr.edu.au; Shu Kun Yang - shukunyang@163.com; Philip S Craig - p.s.craig@salford.ac.uk; Donald P McManus* - donM@qimr.edu.au

* Corresponding author

Published: 4 November 2009

Parasites \& Vectors 2009, 2:53 doi:10.1186/1756-3305-2-53
Received: 14 October 2009

Accepted: 4 November 2009

This article is available from: http://www.parasitesandvectors.com/content/2/I/53

(c) 2009 Yang et al; licensee BioMed Central Ltd.

This is an Open Access article distributed under the terms of the Creative Commons Attribution License (http://creativecommons.org/licenses/by/2.0), which permits unrestricted use, distribution, and reproduction in any medium, provided the original work is properly cited.

\begin{abstract}
During analysis of retrospective community survey data, we identified two patients from Xiji County, south Ningxia Hui Autonomous Region with simultaneous echinococcosis and tuberculosis (TB), representing the first such reports for China. As the echinococcosis chronicity increased, the immune profile in both subjects changed from a ThI to Th2 response, as shown by a TB skin test, originally positive, becoming negative. Such an elevated Th2 immune profile, with subsequent suppression of the ThI immune response, is a common feature of chronic helminth infections. Given the difficulties in definitive diagnosis, and the potential increased susceptibility for TB infection in patients with advanced echinococcosis, we suggest that combined TB/ echinococcosis surveys be undertaken in this area in the future. This would allow early diagnosis of both TB and echinococcosis cases with better prognosis for effective and sustainable treatment outcomes, ultimately reducing associated morbidity and mortality, and also the overall financial costs to the individual and the public health care system in this under developed part of China.
\end{abstract}

\section{Findings}

Tuberculosis (TB) affects over five million people in China, 80 percent living in rural areas [1]. Community surveys in 1988-89 in rural townships of Xiji County in south Ningxia Hui Autonomous Region (NHAR), northwest China indicated a TB prevalence ranging from 0.6$12.4 \%$ (mean 2.2\%) [2]. Helminthiases are also extremely common in China [3]. In Xiji, the prevalence of echinococcosis ranges from $0-7.4 \%$ (mean $2 \%$ ) and from $1-8.1 \%$ (mean 2.5\%) for the cystic (CE) and alveolar AE forms, respectively [4]. Given the similarities in their morbidities, specific diagnosis of TB and echinococcosis in co- infected individuals is difficult which may account for the limited number of reports of simultaneous infections globally [5].

Retrospective community survey data for $\mathrm{TB}$ and echinococcosis $[2,4]$ revealed co-endemic TB and echinococcosis in a number of townships within Xiji County. The range of TB prevalence $(0.6-12.4 \%)$ in the $\mathrm{TB} /$ echinococcosis co-endemic townships was similar to that of the entire county [2] but the mean prevalence $(3.9 \% \mathrm{v} 2.2 \%)$ was significantly higher (Chi-square; $\mathrm{P}=0.036$ ). Prevalence of echinococcosis in the townships ranged from $0.8 \%-9 \%$ 
(mean 4.5\%). It is noteworthy that while the surveys were up to 15 years apart, the incubation period for AE/CE is 10-15 years [6] and so primary Mycobacterium tuberculosis and Echinococcus infections would have been occurring at the same time.

We describe two survey subjects with simultaneous echinococcosis and $\mathrm{TB}$, representing the first such reports for China. Both subjects indicated by questionnaire having received Bacillus Calmette-Guerrin (BCG) vaccination during childhood, confirmed by a characteristic raised skin vaccination scar. Approval for the surveys was given by the Ethics Committee of Ningxia Medical College; written consent to participate was obtained from both patients.

The first, an 18-year-old male reported to Xiji County Hospital in 2006 with malaise, weakness, fatigue and a minor cough. Ultrasound (US) and Computed Tomography (CT) scans identified an uneven hypo-echo image with two irregular lesions in the right liver suggestive of P3 stage AE [6] (Fig. 1.1a). Chest x-ray images showed a number of small shadows scattered on both sides of his lungs, the largest being in the lower lobe of the left lung; CT scans showed a defined edge (Fig. 1.1c \&1.1d), possibly a CE lesion. Further US and serology (highly positive for anti-Em18 antibodies [7]) confirmed P3 stage AE in the liver with possible metastasis to the lungs. He was prescribed albendazole (ABZ) ( $10 \mathrm{mg} / \mathrm{kg} /$ day) and advised to attend the annual clinical follow-up thereafter.

The patient had been previously diagnosed with pulmonary TB by chest $\mathrm{x}$-ray in 2000; he had a positive tuberculin skin test and acid fast bacilli were present in his sputum. Clearly, the earlier BCG vaccination did not protect. A routine clinical examination in 2003 indicated that the TB had been cured following three years of regular TB chemotherapy [8]. X-rays taken in 2006 during the Xiji County Hospital visit were inconclusive and further tests to assess TB status were undertaken. A negative tuberculin skin test and sputum test ruled out relapsed TB. At followup in 2008, the tuberculin skin test had reverted, becoming positive. CT scans of the liver showed that the $\mathrm{AE}$ lesion had shrunk markedly in size (Fig. 1.1b) indicating effective ABZ treatment. Serology was marginally positive for anti-Em 18 antibodies. A CT scan of the chest showed a fibre-like scar in the middle right lung and a calcified lymph node in the middle right hilum (Fig. 1.1e). Moreover, the lesion was irregular, with greater density (Fig. 1.1e and 1.1f) compared with the 2006 CT scan (Fig. 1.1c and 1.1d). The clinical diagnosis was confirmed CE in the lung and effective $\mathrm{ABZ}$ treatment.

The second subject was a 36 year-old female referred to Ningxia Teaching Hospital, Yinchuan city in 2003 com- plaining of constant abdominal pain. Her BCG vaccination had not been protective as chest X-rays in 1998 showed TB lesions on both lungs and she had a positive tuberculin skin test but negative TB sputum test. Despite having been prescribed TB drugs [8], that she had taken intermittently, there were concerns that the TB had invaded her abdomen. X-rays of the chest and abdomen were normal and the tuberculin skin and sputum tests were negative. Abdominal CT (Fig. 1.2a) indicated an advanced stage (P4) liver AE lesion with vascular and biliary involvement of the right liver, with scattered calcifications within the lesion. Serology [7] for anti-EgB, but not anti-Em18, antibodies, was positive. She was prescribed $\mathrm{ABZ}$ and followed-up annually by US and questionnaire examination [4].

In 2005, questionnaire analysis revealed she had two recent episodes of severe coughing with mucus and white gelatinous membranous materials expelled, the first in late 2004 following 18 months of ABZ treatment and the second in the spring of 2005. Microscopic examination after the second episode showed the presence of CE-laminated-membranes [9]. The patient was thus diagnosed with simultaneous AE and CE. Continuous ABZ was prescribed with annual follow-up to monitor treatment efficacy.

Chest CT scans in 2008 showed scars on the top and middle lobes of both sides of the lung, calcification on both sides of their related hilar lymph nodes (Fig. 1.2c), and a $5 \times 1.5 \mathrm{~cm}$ density tissue scar in the lower right lobe (Fig. 1.2d), indicating ABZ had affected the CE lesion. A liver CT scan showed the same AE lesion (Fig. 1.2b) recorded in 2003 (Fig. 1.2a), with marginally fewer liquefaction areas than before, indicating ABZ had had limited effect. Em18 serology and the tuberculin skin and sputum tests for $\mathrm{TB}$ were negative.

These are the first cases of simultaneous TB and echinococcosis reported in China; both patients presented with simultaneous $\mathrm{AE}$ and $\mathrm{CE}$ as well, a feature not uncommon for NHAR [10]. The significantly increased TB prevalence in residents of Xiji townships co-endemic for TB and echinococcosis compared with those from townships endemic only for TB, suggested the increase may be due to co-infection with Echinococcus.

As the echinococcosis chronicity increased in the two patients (following the latency period after ingestion of Echinococcus eggs), the immune profile appeared to change from a Th 1 to Th2 response, reflected by the positive tuberculin skin test becoming negative. This corresponded mainly with the progression of AE from early (P1 \& P2) to advanced (P3 \& P4) stage disease [6], as the infiltrative growth of an $\mathrm{AE}$ lesion releases considerably more 

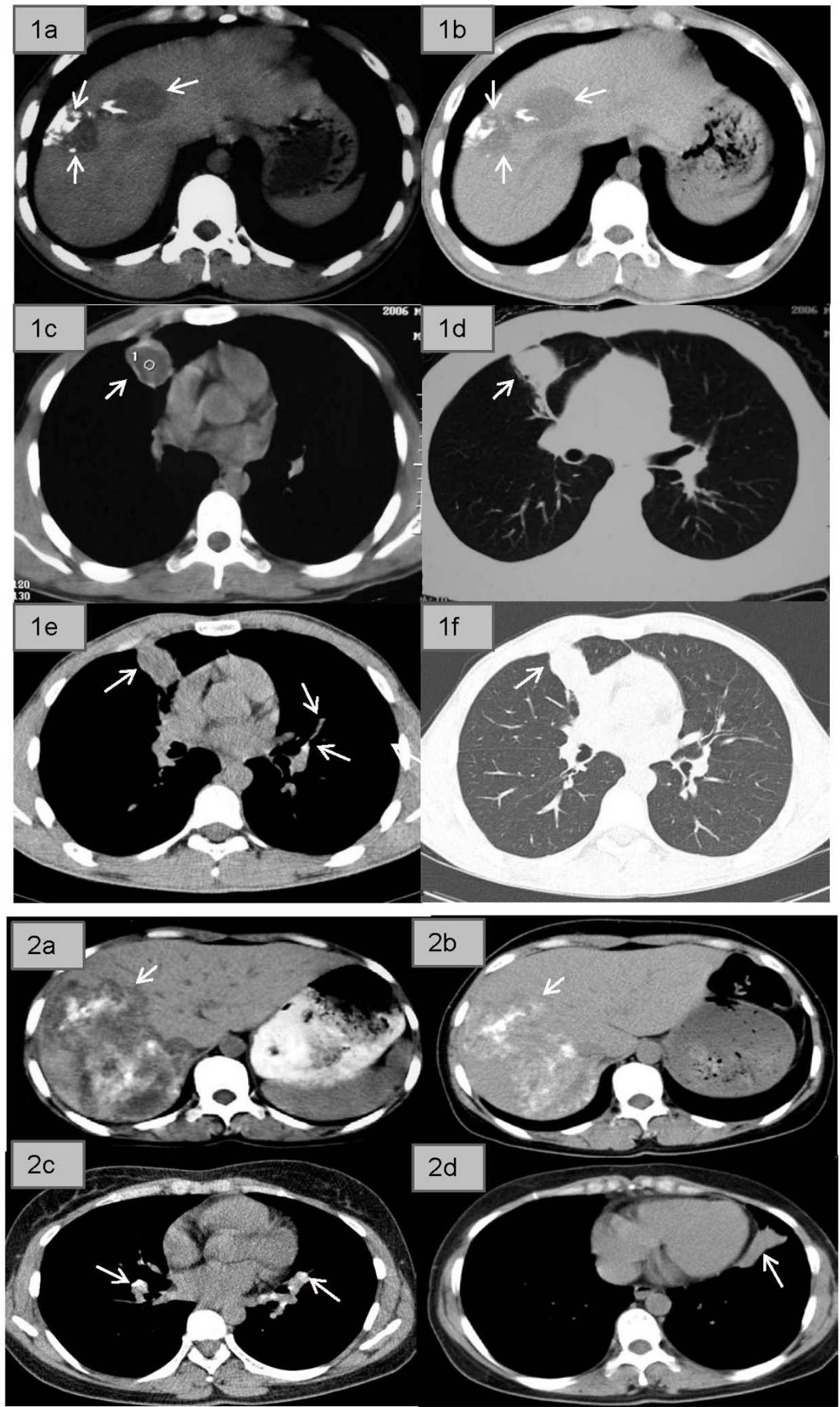

Figure I (see legend on next page) 
Figure I (see previous page)

CT scans of patient I (I 8-year-old male) (panels Ia-f) and patient 2 (36 year-old female) (panels 2 a-d). Panel Ia shows the liver in 2006 showing two irregular lesions located in the right lobe with scattered calcifications. Panel Ib is a followup scan of the liver taken in 2008 showing that the lesions had shrunk in size after treatment with albendazole. Panels Ic and Id show the lung in $\mathbf{2 0 0 6}$ having a lesion with the density of liquid, a clear edge, and surrounding inflammatory infiltrate extending to the anterior pleura. Panels Ie and If are follow-up lung CT scans taken in 2008 showing the lesion had shrunk in size. Panel 2a shows the liver in 2003 with a large irregular lesion located in the right lobe having scattered calcifications and liquefactions within it. Panel $2 b$ shows the liver in 2008 indicating that the same lesion had not changed in size but with increased calcification and decreased liquefaction areas present. Panels $2 c$ and $2 d$ show the lung in 2008 . Panel $2 c$ shows a number of calcified lymph nodes on both sides of the upper and middle lung hila (indicating previously active TB). Panel $2 \mathrm{~d}$ shows scar tissue (approximately $1.5 \times 5 \mathrm{~cm}$ ) in the right lower lobe of the lung without related lymph node calcification.

parasite antigen constantly stimulating the host immune system [6]. An elevated Th2 immune profile and subsequent suppression of the Th1 immune response is common in helminth infections [11]. With a suppressed Th1 immune profile the hosts' ability to detect and respond to viruses, bacteria and other pathogens is impaired [12] and Th1-based vaccines can become ineffective.

Despite BCG vaccination, both subjects developed TB and were skin-test-positive. Following TB treatment, they developed non-specific symptoms resembling a relapsed TB infection; however both cases then became skin testnegative and were subsequently diagnosed with advanced stage (P3 or P4) AE. Given the length of the incubation period of an Echinococcus infection, it is most likely that the cases were infected prior to contracting TB.

Albendazole can vary in its efficacy against echinococcosis, particularly AE [6], exemplified by these two patients. In the first, treatment was effective as shown in 2008 by CT scans of the liver showing regression of the AE lesion, with his immune profile reverting to Th1 as shown by the tuberculin skin test reverting to positive. In contrast, treatment of the female subject was largely ineffectual; the large AE lesion showed no reduction in size and the tuberculin test remained negative.

Similarities in the symptoms of $\mathrm{TB}$ and echinococcosis and the immune progression of both diseases make their definitive diagnosis difficult. A TB infection can often mask the symptoms of early stage echinococcosis, particularly pulmonary echinococcosis. Suppression of the immune system can then promote the progression to later stage echinococcosis or secondary metastases in the case of AE. Moreover, subjects with advanced stage echinococcosis have a pronounced Th2 immune profile [11], mirrored in both study subjects by their negative tuberculin skin test. A previous report of helminth infected Ethiopian immigrants showed they responded poorly to skin testing for TB compared with immigrants who had been dewormed [13] indicating that this immune modulation may compromise the diagnostic efficacy of the tuberculin skin test. Further, studies have also shown that severe AE or CE cases exhibit poor lymphocyte responses both in vivo and in vitro with the Th1 immune response being strongly suppressed in patients with advanced disease $[12,14]$.

A recent study has suggested a mechanism whereby concomitant systemic helminth infections predispose to the development of active tuberculosis in humans [15]. By analysing the cellular responses to mycobacterial antigens in patients who had latent tuberculosis with or without filarial infection, the study demonstrated that filarial infection coincident with $M$. tuberculosis infection significantly diminishes M. tuberculosis-specific Th1 (interleukin [IL]-12 and IFN-gamma) and type 17 T helper (Th17; IL23 and IL-17) responses related to increased expression of cytotoxic $\mathrm{T}$ lymphocyte antigen (CTLA)-4 and programmed death (PD)-1. These findings reinforce our earlier comments regarding vaccine use in helminth endemic countries in that those requiring a Th1 or Th17 response for efficacy may not function optimally in the presence of a chronic helminth coinfection.

Xiji County is underdeveloped and poor and the local hospitals generally lack adequate facilities and technical expertise, with laboratory diagnosis often unhelpful. The majority of TB cases are usually diagnosed by clinical features, exposure history, a positive tuberculin test and the presence of bacteria in sputum by microscopy. This latter method is rapid, but it is relatively insensitive if insufficient sputum is provided, or if few bacteria are present [16]. Given there is co-endemic TB and echinococcosis in Xiji, the difficulties in definitive diagnosis, and the potential increased susceptibility for TB infection in patients with advanced echinococcosis, we suggest that combined $\mathrm{TB} /$ echinococcosis surveys be undertaken in this area in the future. This would allow early diagnosis of both TB and echinococcosis cases with better prognosis for effective and sustainable treatment outcomes, ultimately reducing associated morbidity and mortality, and also the overall financial costs to the individual and the public health care system in this under developed part of China. 


\section{Competing interests}

The authors declare that they have no competing interests.

\section{Authors' contributions}

YRY, PSC and DPM conceived the study, YRY carried out the survey and questionnaire work, SKY undertook the US and CT scans, YRY, DG, ME and DPM analysed the data, and YRY, DG, ME and DPM wrote the manuscript.

\section{Acknowledgements}

We thank Li Yan Bin, Liu Rui Qi and Dominique Vuitton who helped with the community surveys, Akira Ito who undertook the AE/CE serology, and James McCarthy for his helpful comments on the manuscript. Ningxia Medical University provided financial support.

\section{References}

I. Liu J], Yao HY, Liu EY: Analysis of factors affecting the epidemiology of tuberculosis in China. Int J Tuberc Lung Dis 2005, 9:450-454.

2. Xiji County Health Records. Guyuan Prefecture Publishing House; 1990.

3. Chen YD, Tang LH, Xu LQ: Current status of soil-transmitted nematode infection in China. Biomed Environ Sci 2008, 21:173-179.

4. Yang YR, Sun T, Li Z, Zhang J, Teng J, Liu X, Liu R, Zhao R, Jones MK, Wang Y, Wen H, Feng X, Zhao Q, Zhao Y, Shi D, Bartholomot B, Vuitton DA, Pleydell D, Giraudoux P, Ito A, Danson MF, Boufana B, Craig PS, Williams GM, McManus DP: Community surveys and risk factor analysis of human alveolar and cystic echinococcosis in Ningxia Hui Autonomous Region, China. Bull World Health Organ 2006, 84:7|4-72I.

5. Karande SC, Sheth SS, Lahiri KR, Shah MD: Coexistent hydatid disease and pulmonary tuberculosis in a five year old girl. J Assoc Physicians India 1991, 39:353-354.

6. Pawłowski ZS, Eckert J, Vuitton DA, Ammann RW, Kern P, Craig PS, Dar KF, De Rosa F, Filice C, Gottstein B, Grimm F, Macpherson CNL, Sato N, Todorov T, Uchino J, von Sinner W, Wen H: Echinococcosis in humans: clinical aspects, diagnosis and treatment. In WHOI/OIE Manual on Echinococcosis in Humans and Animals: a Public Health Problem of Global Concern Edited by: Eckert J, Gemmell M, Meslin F-X, Pawlowski Z. Paris: World Organization for Animal Health; 200I:20-7I.

7. Ito A, Ma L, Schantz PM, Gottstein B, Liu YH, Chai JJ, Abdel-Hafez SK, Altintas N, Joshi DD, Lightowlers MW, Pawlowski ZS: Differential serodiagnosis for cystic and alveolar echinococcosis using fractions of Echinococcus granulosus cyst fluid (antigen B) and E. multilocularis protoscolex (EMI8). Am J Trop Med Hyg 1999, 60:188-192.

8. World Health Organization: Guidelines for the Management of Drug Resistant Tuberculosis. Publication No. WHO/TB/96. Geneva 1997.

9. Sirlak M, Ozcinar E, Eren NT, Eryilmaz S, Uysalel A, Enneli D, Ozyurda $\mathrm{U}$ : Multiple hydatid cystectomy of the heart necessitating LIMA to LAD anastomosis in a young patient. Cardiovasc Pathol 2009, 18:53-56.

10. Yang YR, Liu XZ, Vuitton DA, Bartholomot B, Wang YH, Ito A, Craig PS, McManus DP: Simultaneous alveolar and cystic echinococcosis of the liver. Trans R Soc Trop Med Hyg 2006, 100:597-600.

II. van Riet E, Hartgers FC, Yazdanbakhsh M: Chronic helminth infections induce immunomodulation: consequences and mechanisms. Immunobiology 2007, 2 I 2:475-490.

12. Kocherscheidt L, Flakowski AK, Gruner B, Hamm DM, Dietz K, Kern P, Soboslay PT: Echinococcus multilocularis: inflammatory and regulatory chemokine responses in patients with progressive, stable and cured alveolar echinococcosis. Exp Parasitol 2008, I I 9:467-474.

13. Elias D, Wolday D, Akuffo H, Petros B, Bronner U, Britton S: Effect of deworming on human $T$ cell responses to mycobacterial antigens in helminth-exposed individuals before and after bacille Calmette-Guerin (BCG) vaccination. Clin Exp Immunol 200I, I 23:219-225.
14. Kacprzak E, Stefaniak J: Evaluating the activity of liver cystic echinococcosis using the delayed-hypersensitivity skin reaction to common antigens. Ann Trop Med Parasitol 1995, 89:25-29.

15. Babu S, Bhat SQ, Kumar NP, Jayantasri S, Rukmani S, Kumaran P, Gopi PG, Kolappan C, Kumaraswami V, Nutman TB: Human type I and 17 responses in latent tuberculosis are modulated by coincident filarial infection through cytotoxic $T$ lymphocyte antigen-4 and programmed death-I. J Infect Dis 2009, 200:288-298.

16. API TB Consensus Guidelines 2006: Management of pulmonary tuberculosis, extra-pulmonary tuberculosis and tuberculosis in special situations. J Assoc Physicians India 2006, 54:219-234.
Publish with Bio Med Central and every scientist can read your work free of charge

"BioMed Central will be the most significant development for disseminating the results of biomedical research in our lifetime. "

Sir Paul Nurse, Cancer Research UK

Your research papers will be:

- available free of charge to the entire biomedical community

- peer reviewed and published immediately upon acceptance

- cited in PubMed and archived on PubMed Central

- yours - you keep the copyright

Submit your manuscript here:

http://www.biomedcentral.com/info/publishing_adv.asp
BioMedcentral 